LA-UR-01-1917

Approved for public release: distribution is unlimited.

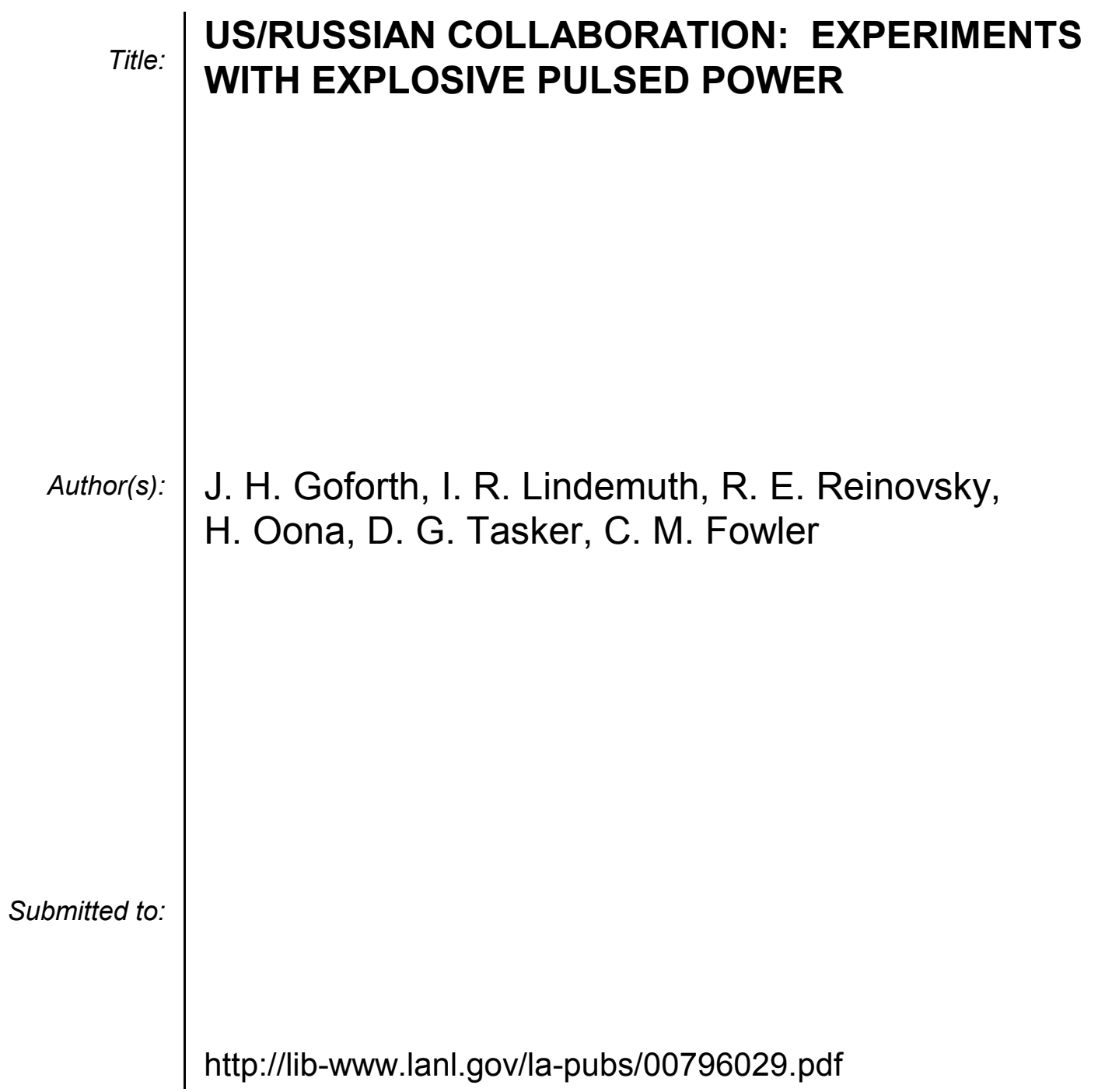

Los Alamos National Laboratory, an affirmative action/equal opportunity employer, is operated by the University of California for the U.S. Department of Energy under contract W-7405-ENG-36. By acceptance of this article, the publisher recognizes that the U.S. Government retains a nonexclusive, royaltyfree license to publish or reproduce the published form of this contribution, or to allow others to do so, for U.S. Government purposes. Los Alamos National Laboratory requests that the publisher identify this article as work performed under the auspices of the U.S. Department of Energy. Los Alamos National Laboratory strongly supports academic freedom and a researcher's right to publish; as an institution, however, the Laboratory does not endorse the viewpoint of a publication or guarantee its technical correctness. 


\title{
US/RUSSIAN COLLABORATION: EXPERIMENTS WITH EXPLOSIVE PULSED POWER
}

\author{
J. H. Goforth, I. R. Lindemuth, R. E. Reinovsky, H. Oona, D. G. Tasker, \\ C. M.. Fowler \\ Los Alamos National Laboratory
}

\section{Introduction}

Through conferences and technical publications, personnel at Los Alamos National Laboratory (LANL) and the All-Russian Institute of Experimental Physics (VNIIEF) learned of each other's interests in both high explosive pulsed power (HEPP) systems and their applications to fusion and high magnetic field research. The principle forum for contact was the Megagauss (MG) Conference series, becoming visible in 1979 at the MG-II Conference in Washington D. C. and culminating in Novosibirsk at MG-V in 1989. Conversations at MG-V led to a proposal, advanced by VNIIEF in 1991, to form a collaborative research agreement. After further preliminary conversations, a contingent from VNIIEF arrived at the MG-VI conference in Albuquerque in 1992 with plans to visit Los Alamos after the conference and finalize an agreement. The two laboratories signed a Memorandum of Understanding (MOU) at this time, November of 1992, agreeing to conduct joint experiments using at least two HEPP systems developed by VNIIEF. Since that time, joint experiments have been conducted both at LANL and VNIIEF facilities using a variety of HEPP systems. On a few occasions, the effort has focused on the HEPP system itself, but more often it has focused on scientific applications of mutual interest.

One major focus of the collaboration has been on disk electromagnetic generator (DEMG) systems developed at VNIIEF. To date, experiments demonstrating DEMG performance at diameters of $0.4 \mathrm{~m}$ and $1 \mathrm{~m}$ have been conducted. DEMG's of $0.4 \mathrm{~m}$ diameter have been further applied to two additional topics. Beginning in 1980 , Los Alamos developed HEPP systems to power plasma z-pinch experiments with the goal of generating $\sim 1 \mathrm{MJ}$ soft $\mathrm{x}$-rays. This work culminated with the Los Alamos Procyon system, which generated 1.7 MJ of soft x-rays in 1995. Recognizing this long term Los Alamos goal (and before Procyon was fully successful), the collaboration challenged VNIIEF to apply their techniques in an innovative way to the soft x-ray production effort. A $0.4 \mathrm{~m}$ DEMG system was chosen for this application. Hydrodynamic liners, for high energy density physics experiments on the Los Alamos Atlas facility, have also been a major focus of the collaboration. Stability experiments to evaluate theoretical capability have been conducted on the Los Alamos Pegasus facility. A 0.4-m VNIIEF DEMG system and the Los Alamos Ranchero HEPP system, have been used to examine Atlas power flow and high energy liner issues at currents up to $30 \mathrm{MA}$.

Another major focus has been on the VNIIEF MC-1 high magnetic field generator. In the 1992 MOU, it was agreed that MC-1 tests would be fielded at Los Alamos as a joint scientific venture. The first series of these tests was fielded in November 1993, and the performance of the MC-1 was demonstrated with Comp B high explosive (HE), a near match to VNIIEF HE, and with Los Alamos PBX-9501. A slightly higher field of $\sim 10 \mathrm{MG}$ was generated in these PBX-9501-driven tests, and the success spawned two additional series of experiments that ultimately brought scientists from six countries together to participate in high magnetic field research 
experiments using VNIIEF hardware at Los Alamos. In addition, the collaboration has coupled Los Alamos and VNIIEF diagnostics, with MC-1 generators at VNIIEF facilities to try to isentropically compress argon into a metallic state.

Magnetized Target Fusion (MTF) has also been a major focus in the collaboration. The VNIIEF MAGO (Russian acronym for a specific MTF configuration) system has been used to generate $10^{13}$ neutrons in a pulse. This system would ultimately provide plasma, with entrained magnetic field, that would be further compressed by a very high-energy liner. An HEPP system developed for the MAGO plasma chamber experiments has been used to field experiments both at VNIIEF and Los Alamos, with a variety of diagnostics from both facilities. In addition, our 1-m DEMG experiment powered a large imploding liner of the type that could ultimately be needed for final plasma compression.

Many aspects of this work have been published in greater detail than is possible here, and we cite the IEEE Transactions in plasma science, ${ }^{1}$ which also includes extensive references. In this paper, we will make a few brief comments about the HEPP systems.

\section{DEMG SYSTEMS}

At the MG-V conference, Chernyshev, and others, ${ }^{2}$ discussed a system, consisting of a multi-stage DEMG and a fuse opening switch, for experiments in the range of several tens of MA with $\sim 1-\mu$ s risetime. This system was one of the two topics specified to be studied in the original MOU. An illustration of the system is shown in

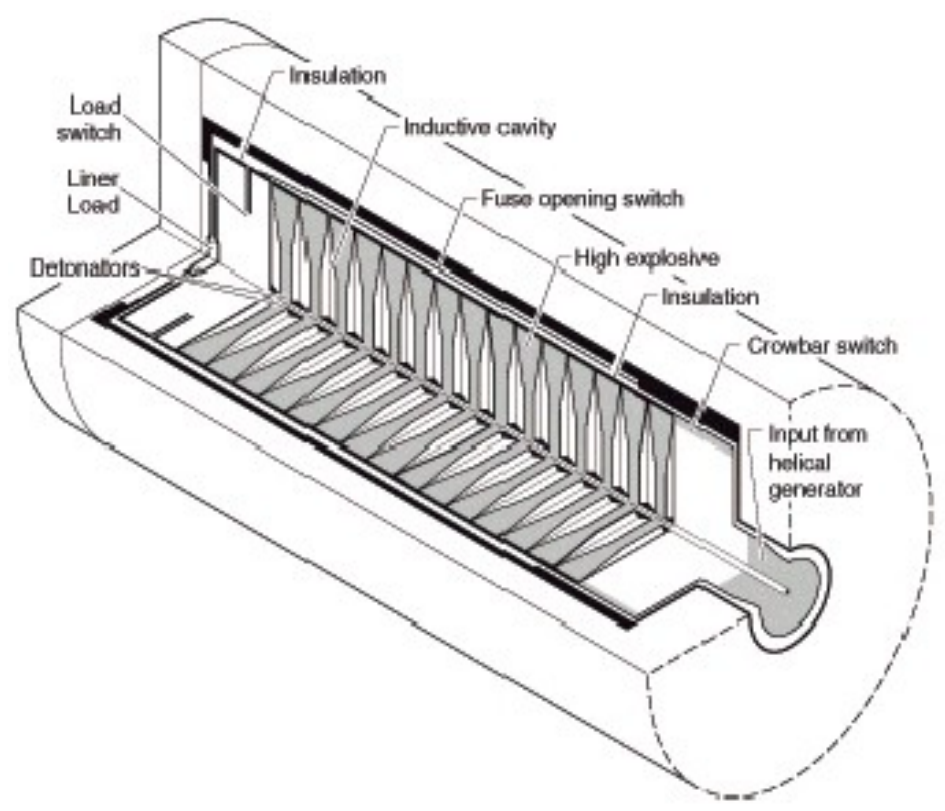

Fig. 1. Illustration of a many-stage DEMG system, with a passive fuse opening switch, and an imploding hydrodynamic liner load. Not shown is the helical generator that provides the initial field for the DEMG. fig. 1. For our first test of the collaboration, we chose to use this system to drive a hydrodynamic liner load, as shown, with enough diagnostics to demonstrate the velocity achieved in the implosion. This test was fielded in September of 1993, and was an historic event as the first time that two nuclear weapons design laboratories from the U. S. and Russia had fielded a joint experiment. Fig. 2 is a group photo of the participants of this test, standing next to the experimental assembly.

The event is even more remarkable, considering that it took place on a firing point previously used for Russian nuclear weapons development, at a facility not known to exist prior to 1990. In the experiment, the DEMG generated $45 \mathrm{MA}$, and $20 \mathrm{MA}$ was delivered to the liner. Pre-shot predictions indicated that $25 \mathrm{MA}$ would be delivered to the load, but the fuse voltage (approaching 500KV), caused a transmission line 


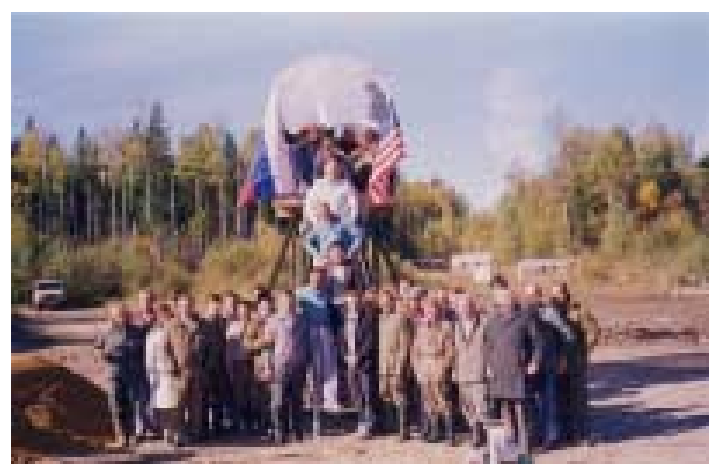

Figure 2. Group photo of the first test of the collaboration, Sept. 1993 at VNIIEF.

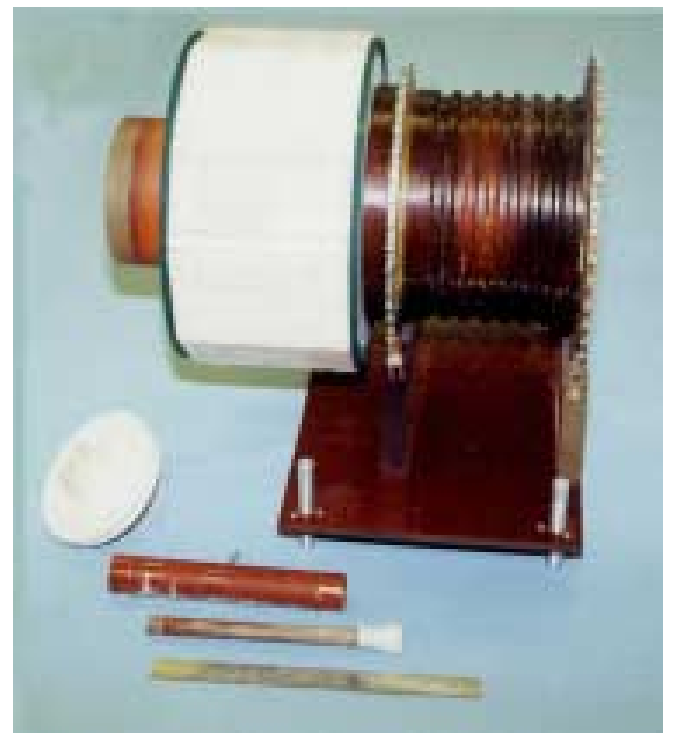

Fig. 3. VNIIEF MC-1 High Magnetic field generator. The two inner cascades and a 30 $\mathrm{cm}$ ruler are in the foreground. failure and not all current went to the load. For our first joint venture, we felt that we had achieved a remarkable success. Subsequent tests have generated currents as high as $60 \mathrm{MA}$ with this system, and $170 \mathrm{MA}$ with the 1-m diameter system.

\section{MC-1 Experiments}

Figure 3 is a photograph of a VNIIEF $\mathrm{MC}-1$ generator, and Fig. 4 shows the magnetic field curves generated in the working volume using Comp B and

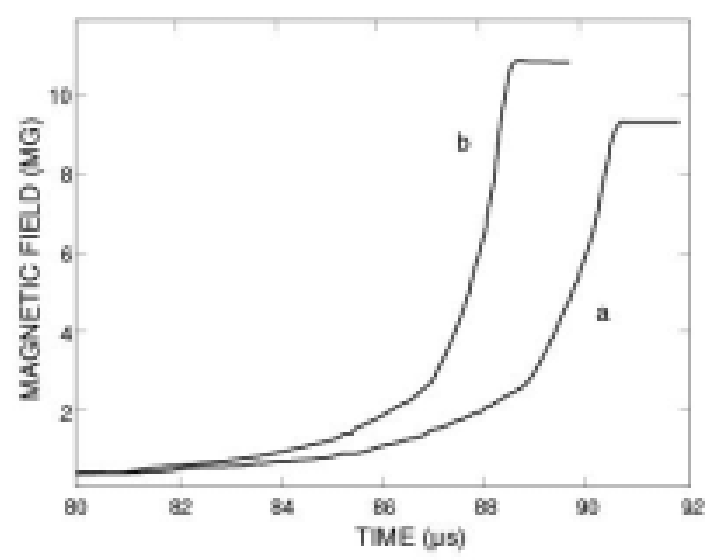

Fig. 4. Magnetic field generated in working volume of MC-1 generator using a) Comp B and b) PBX-9501 HE.

PBX-9501 HE respectively. PBX-9501 is a higher energy explosive than Comp B, which is similar to the VNIIEF explosive

used in the development of the MC-1 devices. Experiments were required to ascertain that the higher energy HE would actually enhance performance, since the MC-1 was optimized for the other HE. The MC-1 operates by injecting magnetic field, via current from a capacitor bank, through a solenoid consisting of fine wires cast into a plastic. Once the field is established inside the generator, the HE shocks the solenoid, which fuses into a conducting cylinder and implodes, compressing the field. At the position where the cylinder begins to become unstable, it collides with a second cylinder similarly constructed. Again, the field penetrates the non-conducting matrix, but the shock wave causes the cylinder to conduct, trapping the field and further compressing it. For the highest fields, a third cascade is used, and experiments are conducted in a few cubic millimeter volume along the axis of the device. Los Alamos scientists first became aware of this system at the MG-5 conference. ${ }^{3}$ 


\section{Magnetized Target Fusion}

The concepts involved in the MTF schemes we have worked on in this collaboration

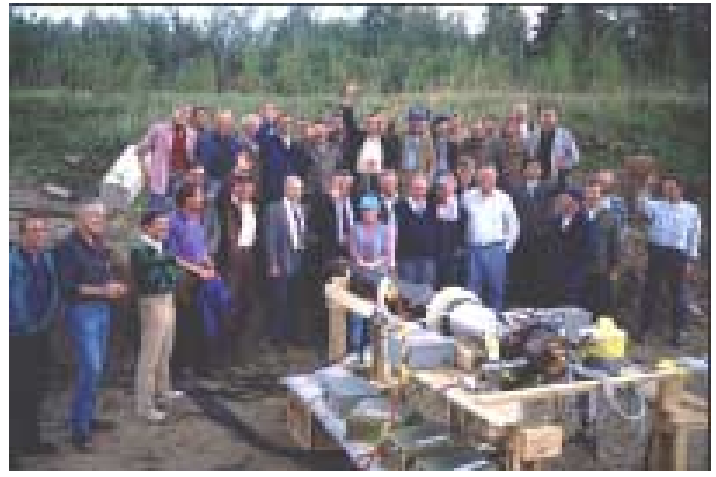

Fig. 5. Group photo of 1996 MAGO test at VNIIEF. The system consists of a helical generator, a storage inductor and opening switch, and a plasma chamber, which is nearest the camera. require considerable explanation, and a detailed description is beyond the scope of this paper. A good description is given in ref.1. For this paper, we picture the primary experimental apparatus in fig. 5. The system uses a helical explosive generator to amplify current from a capacitor bank and deliver some of the magnetic field to a plasma chamber and the rest to a storage inductor. An explosive-opening switch then produces a voltage spike (necessary to the plasma formation) and subsequently causes current from the storage inductor to be transferred to the plasma chamber. Peak current in the load- during the experiment is about 8

MA. A hot plasma with a nearly $10 \mu$ s lifetime is created in the chamber. Compressing a plasma of this type with an imploding liner could lead to fusion.

\section{Conclusion}

In 1992, we began a collaboration between the nuclear weapons design laboratories at Los Alamos and VNIIEF. We have studied topics of mutual interest to and unclassified in both laboratories. We have performed the necessary theoretical work to design and understand these experiments, and have fielded tests at Los Alamos and VNIIEF facilities, sharing diagnostics depending on the capabilities of the laboratories. More technical detail is provided in the referenced papers.

\section{Acknowledgments}

The authors wish to acknowledge the contributions of Vladimir Chernyshev, Alexander Bykov, and Vladislov Mokhov, and the personnel in their departments who have contributed so much to our collective technology and this collaboration.

\section{References}

1. I. R. Lindemuth et. al., U. S./Russian Collaboration in High-Energy-Density Physics Using High-Explosive Pulsed Power: Ultrahigh Current Experiments, Ultrahigh Magnetic Field Applications, and Progress Toward Controlled Thermonuclear Fusion, IEEE Transactions on Plasma Science, Vol. 25, No. 6, December 1997, pp.1357.

2. V. K. Chernyshev, et. al., Investigation of Electrically Exploded Large Area Foil For Current Switching, Megagauss Fields and Pulsed Poser Systems, V. M. Titov and G. A. Shvetsov eds., Nova Science Publishers, New York, 1990, pp. 465.

3. A. I. Pavlovskii, et. al., Production of $15 \mathrm{MG}$ Magnetic Fields in Cascade Ultrahigh Field Generators (MC-1), Megagauss Fields and Pulsed Poser Systems, V. M. Titov and G. A. Shvetsov eds., Nova Science Publishers, New York, 1990, pp. 29. 